\title{
Design of Portable Environment Monitoring System of Ammunition Depots Based on IOT
}

\author{
Jianfeng $\mathrm{Li}^{\text {a }}$, Mingqing Xiao, Haizhen Zhu, Ruoan Wang and Weiwen Shi \\ College of Aerospace Engineering, Air Force Engineering University, Xian 710038, China \\ a1506556759@qq.com
}

Keywords: Environment monitoring system; Portability; IOT; Wi-Fi communication

\begin{abstract}
Aiming at the fact that present environment monitoring systems of ammunition depots have costly wire arrangement, remote monitoring, low reliability and are not able to monitor environment data timely. In this paper, sensor nodes with the core of STM32F103VE chip was designed and the Wi-Fi transmission module with the core of 88 W8686 chip was built. Java is used to develop the intellectualized mobile terminal in eclipse environment. The monitoring terminal is timely and portable which is attained by communication among senor nodes, and the data monitoring center (DMC) and mobile terminal are designed based on Internet of Things (IOT) technology. The relative experiment to test monitoring accuracy and reliability is designed in this paper. The result shows that the monitoring system performs well in monitoring accuracy, communicating reliability and system stabilization. The system has much significance to improve the routine maintenance efficiency of the ammunition depots.
\end{abstract}

\section{Introduction}

Ammunition depots, which exist in caves and remote areas, are responsible for storage of various ammunition. Environment parameters like temperature, humidity, salt fog, are easy to influence the lifetime of ammunition [1]. Therefore, it,s essential to research on prolonging life of ammunition and ensure the security of ammunition depots. At present, lots of wired cables are used in Air Force and environment parameters in ammunition depots are still measured by manual methods, which waste much manpower, material resources and time, and history data can,t be used well [2]. In view of this, the environment monitoring system based on 485 bus is used in some Air Force army [3], which realizes the automatic real-time measurement. Whereas, it is costly, and remote terminals are Industrial Personal Computers with large-scale which is not easy to move.

In recent years, several leaps are realized in data collection systems with the rocketing development of IOT. Digital bus solves the interaction interference in data long-distance transmission, making the long-distance monitoring possible [4]. Aiming at the contradiction between portability and function of detectors, the portable intelligent detector based on wireless communication is designed. But the intellectualization of the monitoring platform is low and the monitoring data can, $t$ be saved in time [5-6]. The research shows the measurement methods of monitoring systems are converted from wired transmission to wireless transmission, from single-point to multi- point, which solve the problems of interference in long-distance transmission, difficulty of saving data.

In this paper, aiming at the fact that the present environment monitoring systems of ammunition depots have costly wire arrangement, remote monitoring, low reliability and are not able to monitor environment data timely, a kind of environment monitoring system for ammunition depots based on wireless network is designed, and Wi-Fi communication and intelligent mobile terminal are introduced to the system. Sensor nodes with the core of STM32F103VE chip and the Wi-Fi transmission module with the core of 88 W8686 chip are built. Java is used to develop the intellectualized mobile terminal software in eclipse compiling environment and the relative experiment to test monitoring accuracy and reliability is designed. 


\section{Overall Design of the System}

The environment monitoring system of ammunition depots is composed of sensor nodes, a DMC and mobile terminals. The overall design of the system is shown in Fig.1. The depot model is set up in DMC, and the arrangement method of fire disaster monitors [7] is introduced to calculate the optimal program of sensor nodes distribution. Sensor nodes are responsible for the collection of environment parameters and pack data to monitoring center through Wi-Fi module. The DMC is designed for data pretreatment, classification and storage, and data results are compressed as packages transferring to mobile terminal by Wi-Fi technology. Workers can use the client to send command anywhere under the coverage of wireless network, and data information will be packed and sent from DMC to the client, and be displayed in the Android mobile terminal.

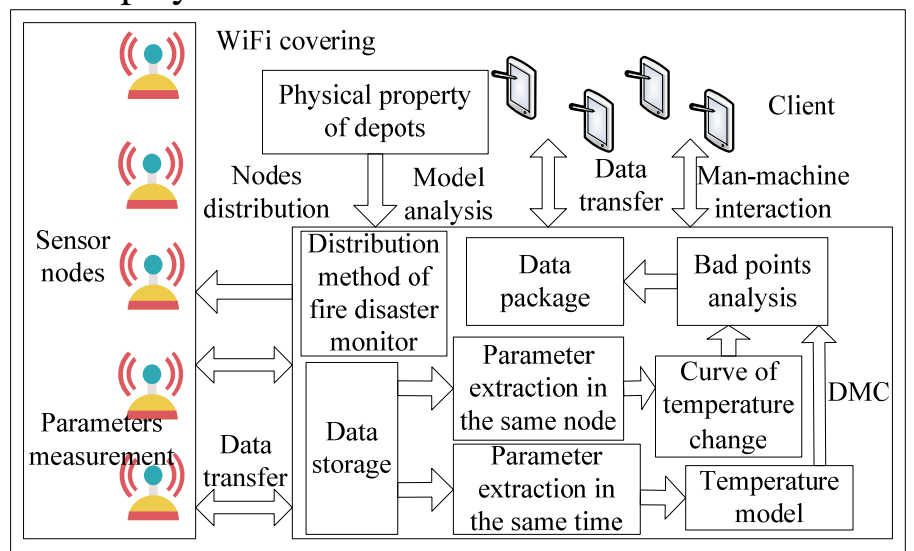

Fig.1 Overall Structure of the system

The measurement modules of temperature, humidity, salt fog and atmospheric pressure is built based on STM32F103VE single chip microcomputer (SCM) in the sensor nodes. The 88W8686 chip from Marvell company is chosen to build Wi-Fi transmission module and realizes the transmission of environment data. Data processing is handled by SQL Server database and Visual Studio software in DMC, and wireless transmission module is added for interaction of sensor nodes and client. Android mobile terminal is chosen as the client carrier to set up upper computer. B/Browser/Server, is adapted to realize the man-machine interaction [8].

\section{Hardware and Software Design of the System}

\subsection{Hardware Design of the System}

Considering the limitation of this paper, the design of temperature sensor module is proposed and the other modules are similar. As the core component of signal collection, LM35DZ temperature sensors have a weak signal output with much noise. So signal denoising and amplification are essential during data collection. The LM324D operational amplifier is introduced in the system, and the magnification is controlled by RW1 in Fig.2.

Considering the difference of supply voltages among signal conditioning circuit module, master control chip module and wireless communication module, WRB2405CS-3W, WRA2415CS-3W and SPX1117 chips are introduced for voltage conversion. Relative circuits of power supply are shown in Fig.3. The alternating current of $220 \mathrm{~V}$ is converted to the direct current of $5 \mathrm{~V}$ by the transformer. The direct current of $5 \mathrm{~V}$ is converted to the direct current of $+5 \mathrm{~V}, \pm 15 \mathrm{~V}$ for the power supply of external devices and amplifying circuits. The direct current of $5 \mathrm{~V}$ is converted to the direct current of 3.3V for the power supply of SCM STM32F103VE by SPX1117 chip. Every supply circuit adds a module of isolation, stabilization and short circuit protection to avoid the load damage from power supply problems. 


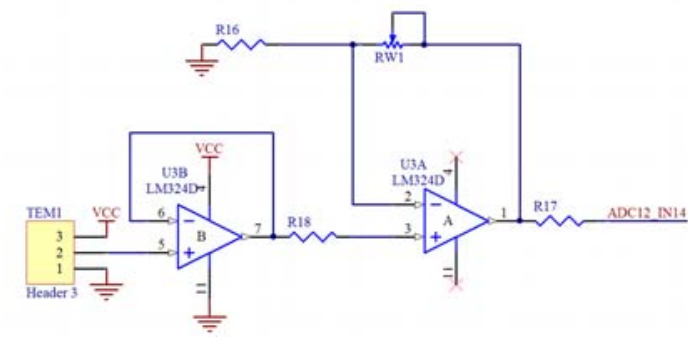

Fig.2 Signal modulating circuit

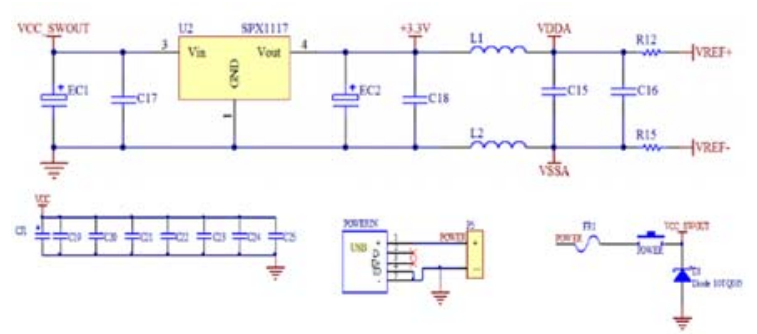

Fig.3 Power supply circuit

As the core of the master control module, STM32F103VE chip is introduced due to its high performance. This chip uses RISC kernel of ARM Cortex-M3 with 32 bit. The working frequency reaches up to $72 \mathrm{MHZ}$, and $128 \mathrm{~KB}$ flash memory is inserted. The abundant I/O ports and the peripheral, which is connected to APB bus, ensure the quick response and provide the prerequisite of expansion and update for peripheral. On the basis of the minimal system of SCM, the main task of the master control module is to set up the platform for signal collecting and conditioning circuit module and wireless communication module. The minimal system of SCM is shown in Fig.4.

Wireless communication module is used to send test data and receive transfer instruction, which is realized by Wi-Fi communication of 88W8686 chip. The design of Wi-Fi circuit is shown in Fig.5. By the connection between Host unit and SDIO port, data information can be sent to the server and data process, allocation, display will be handled further.

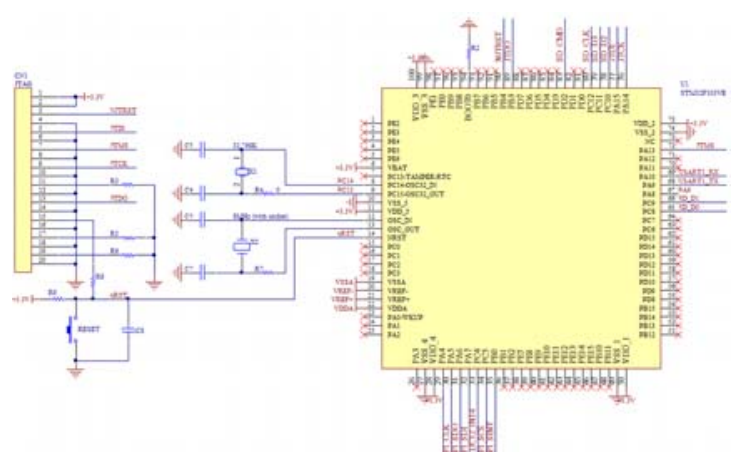

Fig.4 Minimum System of MCU

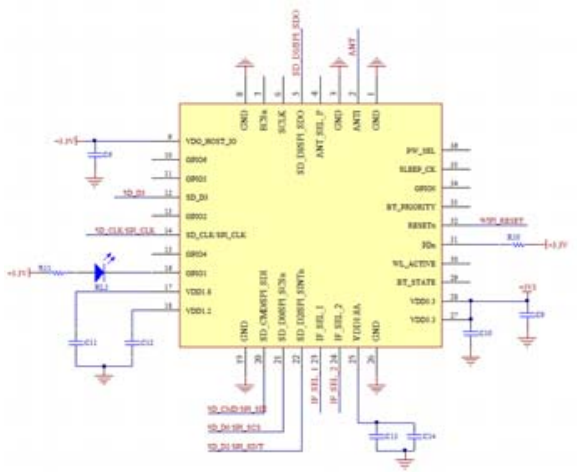

Fig.5 Hardware circuit of Wi-Fi

\subsection{Software Design of the System}

The software design of the system can be divided into two parts: the software design of sensor nodes and data monitoring center.

For sensor nodes, the design before data requirement is shown in Fig.6. Initialization of the whole system is essential such as STM32F103 chip, GPIO, DMA, AD. Then the peripheral is started to collect data and Wi-Fi module is prepared to connect network. If the network connects successfully, a socket will be built, and if not, the requirement of network connection will be continued. After finishing these preparation work, CPU will get into dormancy and the peripheral will collect data normally.

When data requirement happens, data acceptance interruption of the serial port will appear and data transmission will be started. The software design after the data requirement is shown in Fig.7. After the command of data requirement is received by sensor nodes, the work of peripheral such as DMA and AD will be stopped to avoid confliction during data transmission of DMA. After finishing data transmission, the socket connection will be broken, and the peripheral will restart and work normally. CPU gets into dormancy. 

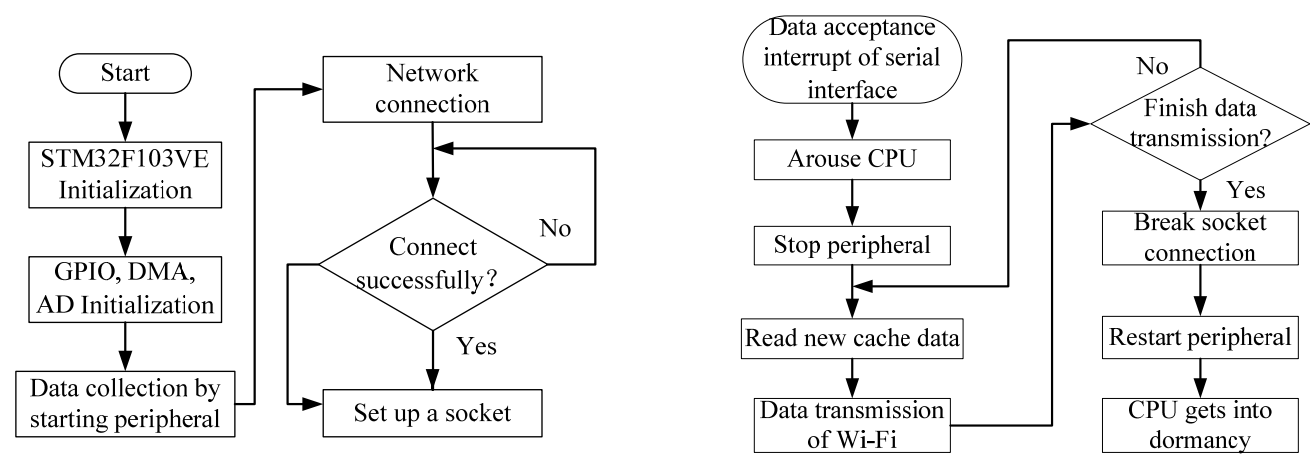

Fig.6 Software design of sensor nodes (Before) Fig.7 Software design of sensor nodes (After)

Data monitoring center is the neural center of the environment monitoring system. $\mathrm{B} / \mathrm{S}$ structure is adapted during the software design. Data is saved by SOR server and database is visited by ADO.NET technology [9]. The software design of data monitoring center is shown in Fig.8. When there is something wrong with the ammunition depot, monitoring parameters from sensor nodes will exceed the safety range and generate an alarm. When bad pots appear, the theory of Kalman Filtering and singular value will be introduced for data analysis, and judge the source of the problem. Then, a decision will be made that whether send the alarm signal to client or not.

For this environment monitoring system, clients only need to use mobile equipment to connect local area network, which is convenient and portable. Then the browser page can be visited to monitor the environment parameters change of the ammunition depot.

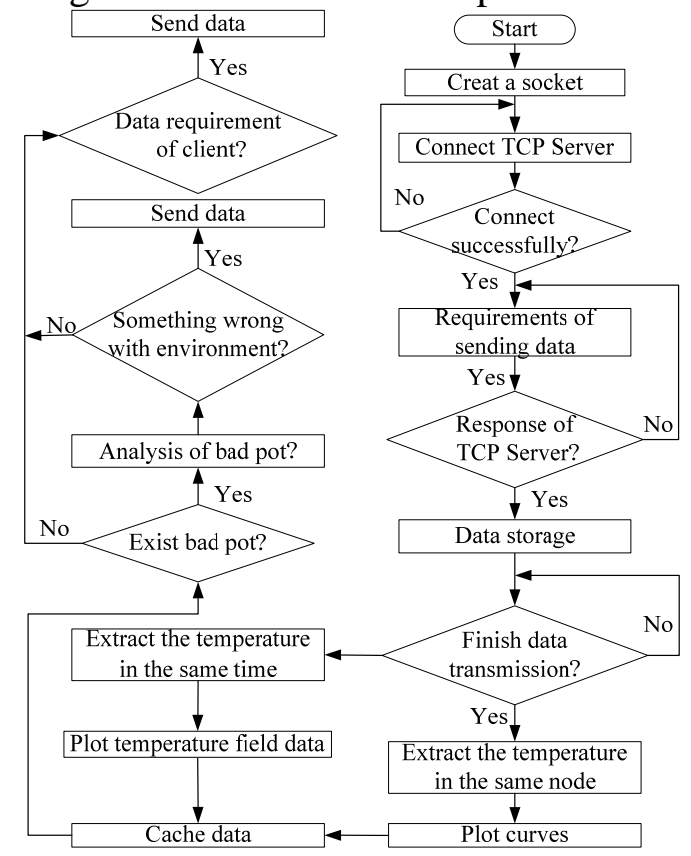

Fig.8 Software design of data monitoring center

\section{Realization and Test of the System}

The experimental board for data collection and processing is shown in Fig. 9. This board is composed of power supply module, serial port module, signal collection and processing module, main control module and Wi-Fi transmission module. 


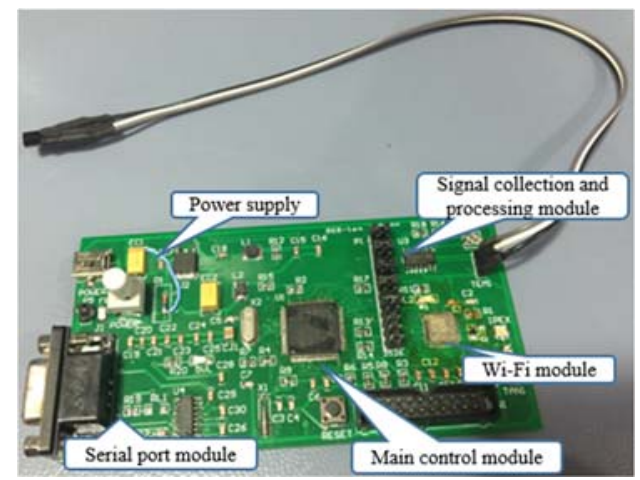

Fig.9 Experimental board of monitoring system

W-Fi connection display and temperature monitoring display of mobile terminal based on Android development environment is shown in Fig. 10 and Fig.11. Operators only need to input IP number and port number. Then parameters like temperature, humidity, salt fog in the ammunition depot can be monitored. The portable environment monitoring system of ammunition depots based on Wi-Fi is debugged successfully. Environment monitoring can be realized in the area of Wi-Fi covering.

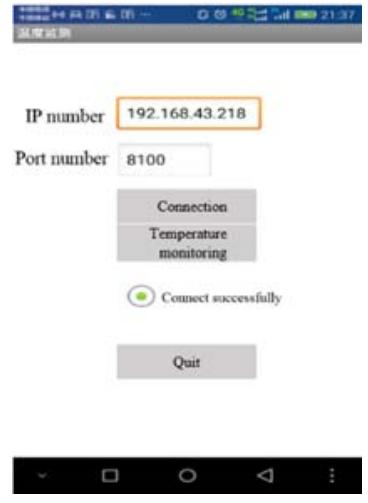

Fig.10 Wi-Fi connection

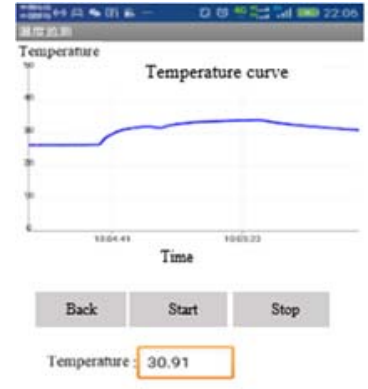

Fig.11 Temperature monitoring

A sequential command and data transmission test are continued for the intelligent monitoring system. In a period of time, data requirements are received from measurement center to data processing center. Requirement times and data transmission successfully times are recorded, which is shown in the Table 1 . After the client send requirements to data processing center, failure of data acceptance may occur due to the connection problem of network. But the failure rate below 0.5\% based on the test, which can be ignored. The test result proves the intelligent system has high reliability.

Tab 1 Test result of communication reliability

\begin{tabular}{cccc}
\hline Monitoring times & Requirement times & Acceptance times & Failure rate of requirements \\
\hline 1 & 1011 & 1009 & $0.19 \%$ \\
2 & 1025 & 1022 & $0.29 \%$ \\
3 & 1023 & 1019 & $0.39 \%$ \\
4 & 1014 & 1011 & $0.30 \%$ \\
5 & 1022 & 1017 & $0.49 \%$ \\
6 & 1029 & 1023 & $0.58 \%$ \\
7 & 1014 & 1010 & $0.39 \%$ \\
8 & 1027 & 1023 & $0.38 \%$ \\
9 & 1037 & 1034 & $0.28 \%$ \\
10 & 1009 & 1004 & $0.49 \%$ \\
\hline
\end{tabular}

To test the monitoring precision of the system, two temperature sensor nodes, two humidity sensor nodes, two salt fog sensor nodes, two atmospheric pressure sensor nodes and instruments with high precision to measure these parameters are put in every three ammunition depot. By Android mobile phones, extracting these parameters data, on average, three hours a time, and make a comparison with the result measured by operators. The result is shown in Fig.12. Based on the test result, this system has features of quick response, high test precision. The test error is controlled within $1.2 \%$ on average, which can basically match the requirement of system design. 


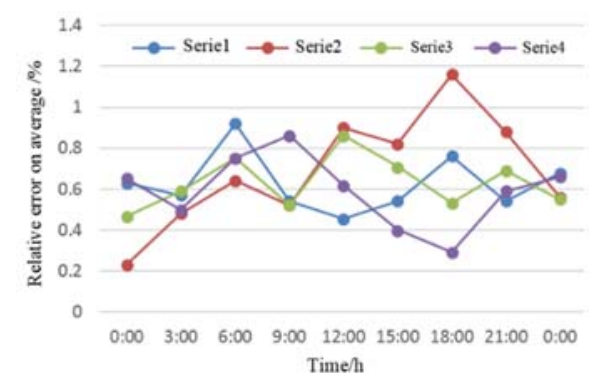

Fig.12 System deviation of environment monitoring

\section{Conclusion}

Aiming at the fact that the present environment monitoring systems of ammunition depots have costly wire arrangement, remote monitoring, low reliability and are not able to monitor environment data timely, an environment monitoring system of ammunition depots based on wireless network is designed, and Wi-Fi communication and intelligent mobile terminal are introduced to the system. Sensor nodes with the core of STM32F103VE chip is designed and the Wi-Fi transmission module with the core of 88 W8686 chip is built. Making use of the Wi-Fi wireless transmission technology and Android environment, a portable, precise, timely monitor based on mobile terminal in the area of network covering is realized in ammunition depots. This is a networked data collection and monitoring system with low cost and high reliability, which has significance to improve the work efficiency in army.

\section{References}

[1]. GAO Yulong, YI Jianzheng, WANG Haidan. The Effects of Storage Environments to Ammunition Quality [J]. Equipment Environment Engineering, 2010, (75): 77-105.

[2]. WEI Chao. The Research of Temperature and Humidity Monitoring System in Army Ammunition [D]. Chengdu, Southwest Jiaotong University, 2010.

[3]. LOU Puyin, SHI Qiong, ZGANG Jing. Design and Achievement of Temperature and Humidity Monitoring System in Army Depots [J]. Microcomputer Information, 2011, 27 (8): 66-67.

[4]. LU Chao. Design of Distributed Temperature Collection System Based on PC and Single Chip Microcomputer [J]. Instrumentation Technology and Sensors, 2007 (6): 35-37.

[5]. FAN Cheng Lin, LIU Yunsheng, ZHOU Baochang, et al. Portable and Wireless Intelligence Temperature Recorder [J]. Instrumentation Technology and Sensors, 2014 (5): 10-12.

[6]. LI Jianwen, ZHENG Yi, WANG Yue, et al. Design of long-distance Temperature Monitoring System Based on Wireless Technology [J]. Instrumentation Technology and Sensors, 2014(2): 68-70.

[7]. ZHANG Guoshu, YUAN Shujie. A New Method to Ensure Arrangement Distance of Fire Detector [J]. Research on Fire Equipment, 2008 (6): 431-432.

[8]. HAN Yudong. Design and Achievement of MY SQL Database Based on B/S IOT Environment Monitoring System [D]. Tianjin University, 2014.

[9]. ZHANG Weihua, FEI Hongwen. Visual Studio 2015 Advanced Programming [M]. Beijing: Tsinghua University Press, 2016, 5.

[10]. SHI Weiming. Android APP Start and Development [M].Beijing: China Machine Press, 2017, 7 